\title{
Germanium Nanocrystals Embedded in Sapphire
}

Q. Xu ${ }^{\mathrm{a}, \mathrm{b}}$, I.D. Sharp ${ }^{\mathrm{a}, \mathrm{b}}$, C.Y. Liao ${ }^{\mathrm{a}, \mathrm{b}}$, D. O. Yi ${ }^{\mathrm{a}, \mathrm{c}}$, J.W. Ager III' ${ }^{\mathrm{a}}$ J.W. Beeman ${ }^{\mathrm{a}}$, K.M. Yu ${ }^{\mathrm{a}}$, D. C. Chrzan ${ }^{\mathrm{a}, \mathrm{b}}$, E.E. Haller, ${ }^{\mathrm{a}, \mathrm{b}}$

${ }^{a}$ Materials Sciences Division, Lawrence Berkeley National Laboratory, Berkeley, CA 94720, U.S.A.

${ }^{\mathrm{b}}$ Department of Materials Science and Engineering, University of California, Berkeley, CA 94720, U.S.A.

'Applied Science and Technology Group, University of California, Berkeley, CA 94720, U.S.A.

\begin{abstract}
${ }^{74} \mathrm{Ge}$ nanocrystals are formed in a sapphire matrix by ion implantation followed by thermal annealing. Transmission electron microscopy (TEM) of as-grown samples reveals that the nanocrystals are faceted and have a bi-modal size distribution. Notably, the matrix remains crystalline despite the large implantation dose and corresponding damage. Embedded nanocrystals experience large compressive stress relative to bulk, as measured by Raman spectroscopy of the zone center optical phonon. In contrast, ion-beam-synthesized nanocrystals embedded in silica are observed to be spherical and experience considerably lower stresses. Also, in situ TEM reveals that nanocrystals embedded in sapphire melt very close to the bulk melting point $\left(\mathrm{Tm}=936{ }^{\circ} \mathrm{C}\right)$ whereas those embedded in silica exhibit a significant melting point hysteresis around $\mathrm{T}_{\mathrm{m}}$.
\end{abstract}

\section{INTRODUCTION}

Surface effects on nanostructure properties have been extensively studied for the simple reason that a large fraction of atoms reside on the surface in nanostructured materials. In the case of embedded Ge nanocrystals, the interface is particularly interesting because on the one hand, quantum mechanical models predict efficient size-dependent photoluminescence [1], but on the other hand, the reported photoluminescence from embedded Ge nanocrystals have mostly been attributed to oxide defects between the oxide and the nanocrystals[2-3]. In this context, it is important to determine the properties of interface defects and the roles they play in determining the optical properties.

Freestanding nanocrystals have been reported to have a reduced melting point compared to bulk material due to their large surface area to volume ratio [4-6]. In contrast, small melting point elevations and hysteresis behaviors have been observed in some embedded metal nanocrystals systems [7]. The determining factors are the large 
interface fraction and the difference in the interface energies between solid/liquid nanoparticles and the surrounding matrix. We have observed a $250^{\circ} \mathrm{C}$ superheating and $100^{\circ} \mathrm{C}$ undercooling thermal hysteresis of Ge nanocrystals embedded in amorphous silica. [8] This alteration in melting behavior well illustrates the influence the matrix and the interface have on the fundamental properties of nanocrystals. The study presented in this paper aims at comparing the effects of a crystalline matrix (sapphire) on Ge nanocrystals with our previous observations of those of amorphous silica matrix.

\section{EXPERIMENT}

A commercially available single crystalline sapphire substrate with an orientation random very close to a-axis was used as the implantation matrix. Isotopically pure ${ }^{74} \mathrm{Ge}$ nanocrystals were fabricated via selective ion implantation. In order to achieve a similar depth profile of concentration as observed in the silica matrix [9], a multi-energy/dose implantation scheme was chosen: $50 \mathrm{keV}\left(3 \times 10^{15} \mathrm{~cm}^{-2}\right), 80 \mathrm{keV}\left(5 \times 10^{15} \mathrm{~cm}^{-2}\right), 120 \mathrm{keV}$ $\left(6 \times 10^{15} \mathrm{~cm}^{-2}\right)$ and $150 \mathrm{keV}\left(8 \times 10^{15} \mathrm{~cm}^{-2}\right)$. Following implantation, multiple samples were cut and annealed in an $\mathrm{Ar}$ atmosphere for $60 \mathrm{~min}$ at $1200^{\circ} \mathrm{C}$, and were subsequently quenched from the annealing temperature to room temperature under running water.

Raman spectra were obtained using the $488 \mathrm{~nm}$ line of an Ar ion laser operating at $150 \mathrm{~mW}$ in a macroscopic optical setup with $5 \mathrm{~cm}^{-1}$ resolution.

Cross-sectional and plan-view TEM specimens were prepared in conventional ways. Structure information and size distribution were obtained from studies performed in several microscopes: Philips CM200, CM300, and JEOL 3010.

As-grown nanocrystal samples were heated in-situ up to $1000^{\circ} \mathrm{C} \pm 15^{\circ} \mathrm{C}$ in a JEOL 3010 electron microscope operating at $300 \mathrm{kV}$. The heating and cooling runs were conducted in $10^{\circ} \mathrm{C} / \mathrm{min}$ steps using a Gatan $628 \mathrm{Ta}$ single tilt heating holder. The beam current was kept at $8 \mu \mathrm{A}$ above dark current so as to minimize beam heating [5]. Bright field and dark field micrographs were recorded in situ onto a video tape.

\section{RESULTS}

The Raman spectrum obtained from as-grown $\mathrm{Ge}$ nanocrystals is shown in Figure 1. The Raman line is blue-shifted relative to that of the isotopically enriched ${ }^{74} \mathrm{Ge}$ single crystal. The

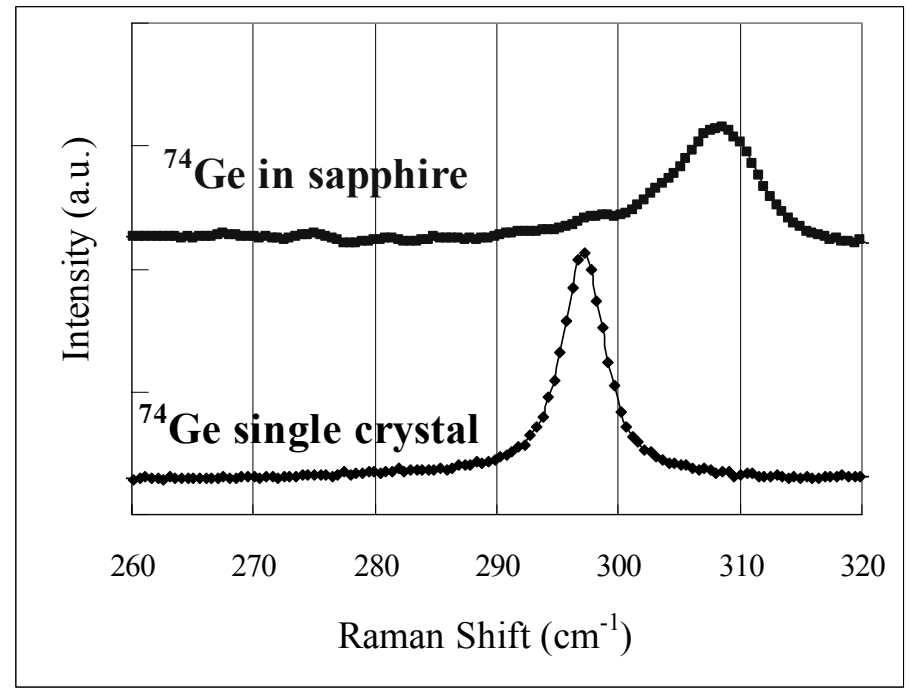

Figure 1 Raman data obtained from 1) as-grown ${ }^{74} \mathrm{Ge}$ nanocrystals embedded in sapphire; 2) ${ }^{74} \mathrm{Ge}$ bulk Ge single crystal. 
shift represents a compressive stress of around $4 \mathrm{GPa}$ determined from a calculation following the model proposed by F. Cerdeira, et al. [10] This big stress can be explained by the $5 \%$ volume expansion Ge nanocrystals that occurs through solidification during the cooling from $1200^{\circ} \mathrm{C}$ growth temperature and the thermal expansion coefficient difference between Ge and sapphire. As a comparison, Ge nanocrystals embedded in silica also exhibit a compressive stress, but with a much lower value of around 1.6GPa.[9] The difference comes not only from the substrates, but also from the fact that the nanocrystals in silica were formed at a lower temperature, $900^{\circ} \mathrm{C}$.

High stress in the nanocrystals is also evident in cross-sectional TEM observations as shown in Figure 2. There are two high contrast areas in the micrograph in Figure 2 a). The line of dark spots deeper beneath the surface shows the end-of-range damage as a result of implantation. The contrast near the surface region comes from the stress field where Ge nanocrystals were formed. A closer look at the highly stressed area is obtained in Figure 2 b) where higher magnification was applied. The white circles enclose some of the nanocrystals visible under this magnification. The "lattice" pattern is not the atomic lattice fringes of the nanocrystals, but the Moiré fringes, observed when two different but similar crystal lattices overlapp each other. The existence of the Moiré fringes indicates that not only are the Ge nanoparticles crystalline; the matrix also remains crystalline surviving the implantation and thermal annealing.

The size distribution of the Ge nanocrystals was obtained through a laborious TEM effort, caused by the high contrast of the stress field that obscured the view of nanocrystals. The cross-sectional TEM specimen was thinned by very low angle ion milling to reduce the contrast of the stress field. Then numerous relatively high magnification micrographs were taken, followed by manually measuring the size of the
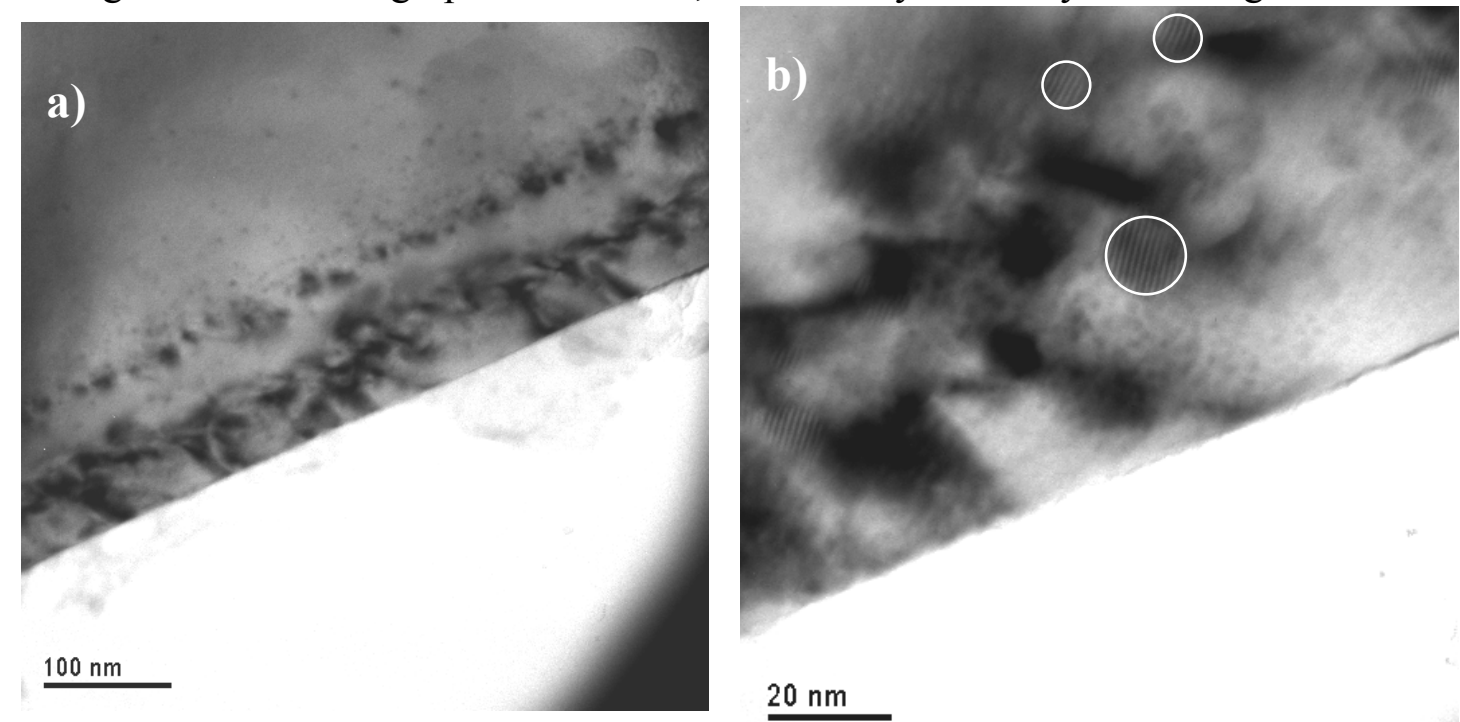

Figure 2 TEM micrograph of a cross-sectional specimen of as-grown Ge nanocrystals embedded in sapphire obtained in a JOEL 3010 at $300 \mathrm{kV}$. a) Lower magnification micrograph shows both stress field and end-of-range damage; $b$ ) higher magnification micrograph shows Moiré fringes of nanocrystals in stress field. 
nanocrystals. The result is shown in Figure 3. The darker bar histogram shows the size distribution of Ge nanocrystals embedded in silica and the lighter bars are the size distribution of nanocrystals in sapphire. The darkest color in the middle shows the overlapping area of the two histograms. The Ge nanocrystals in sapphire are larger on average and have a wider size distribution than those in silica. The larger size is expected since the nanocrystals in sapphire were formed at a much higher temperature than those formed in silica. Second, unlike the near-Gaussian-shaped size distribution of the silica system, the size distribution

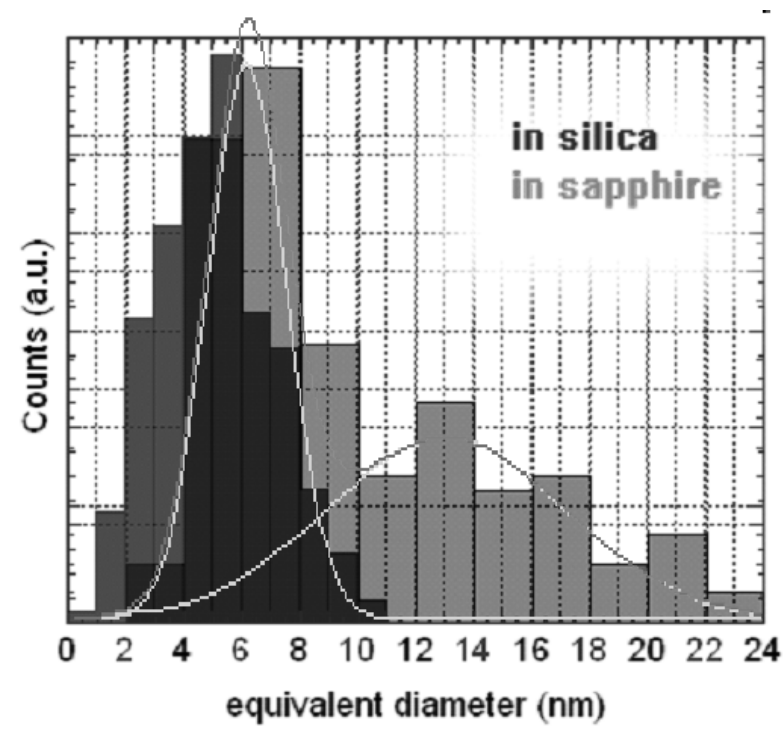

Figure 3 The size distribution of $\mathbf{G e}$ nanocrystals embedded in silica and sapphire. of the sapphire system has a bi-modal shape which can be fitted by two Gaussians very well, corresponding to average diameters of $6.2 \mathrm{~nm}$ and $13.1 \mathrm{~nm}$ respectively. This unusual shape of the size distribution is closely related to and can be well explained by the spatial distribution of the Ge nanocrystals, which is shown in Figure 4.

Figure 4 shows that large sized Ge nanocrystals were formed in one line in the most stressed and damaged area inside the sapphire matrix, where the peak Ge concentration is located after the implantation. The smaller ones are distributed in the shoulder area. This

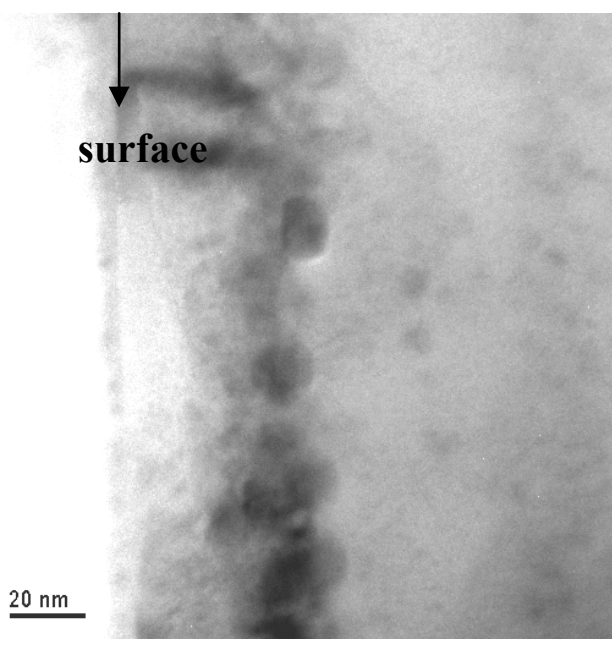

Figure 4 Cross-sectional TEM micrograph shows the spatial distribution of Ge nanocrystals. unique size-related spatial distribution can be attributed to details of Ge diffusion in sapphire. Ge atoms diffuse faster in the more damaged area, where large nanocrystals are thus formed, whereas in the area where Ge atoms are less mobile, smaller nanocrystals are formed.

High resolution TEM micrographs shown in Figure 5 reveal a faceted shape of Ge nanocrystals in sapphire, in contrast to the spherical shape of nanocrystals in silica. This is apparently the result of the anisotropy of the sapphire matrix as opposed to the isotropic amorphous silica matrix. Further analysis of the orientation relationships between the nanocrystals and the sapphire matrix shows that there is no fixed relationship of orientation between the nanocrystals and the 

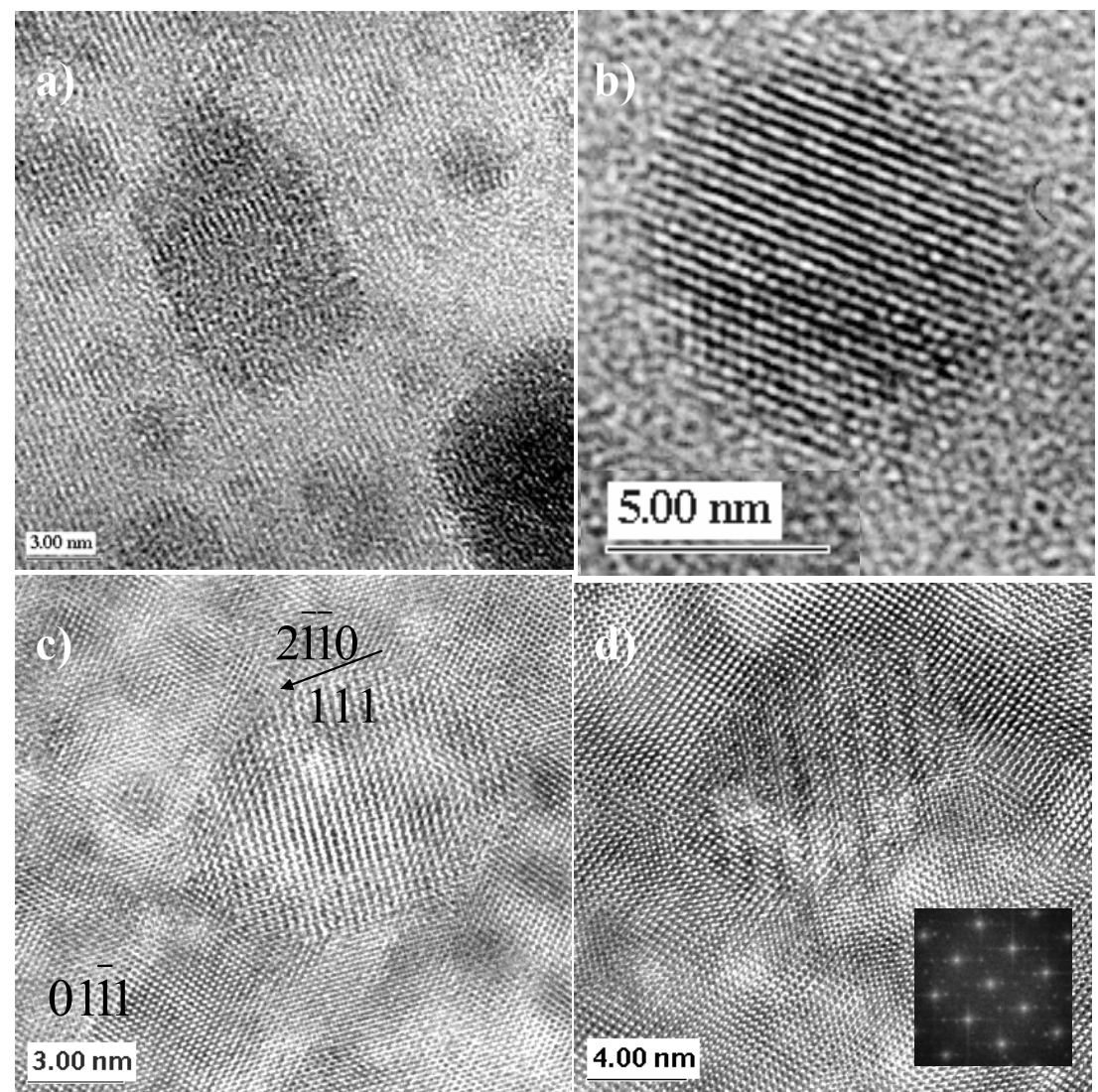

Figure 5 HR-TEM micrographs of Ge nanocrystals: a) embedded in sapphire, plan-view(a-axis of sapphire); b) embedded in silica; c) in sapphire with orientation labeled; d) in sapphire. sapphire. Shown in

Figure $5 \mathrm{c}$ ) is one example of orientations. Different Moiré fringes of different nanocrystals and a powder pattern observed in selective area diffraction are evidence of a random orientation of these nanocrystals.

Similar in-situ heating experiments carried out in the JEOL 3010 electron microscope show very different thermal behaviors of Ge nanocrystals embedded in different matrices. The silica system shows a large melting and solidification

hysteresis around the melting temperature of bulk Ge shown in Figure 6. [8] However the

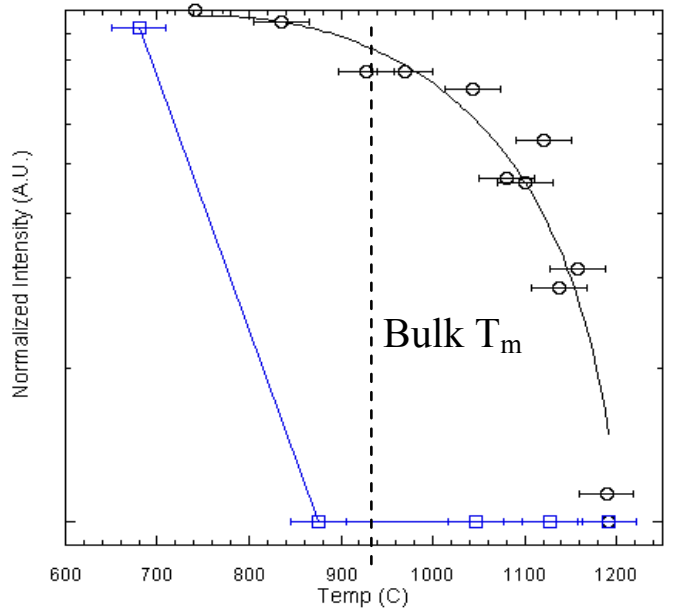

Figure 6 Melting hysteresis loop of Ge nanocrystals in silica drawn from the data extracted from reference [8]. sapphire system shows a bulk like melting behavior. The nanocrystals melt at $955^{\circ} \mathrm{C}$ $+/-15^{\circ} \mathrm{C}$, which is very close to the bulk melting temperature. No definite hysteresis is observed within the measurement capability of the microscope and the holder. The melting is defined by the disappearance of the contrast in the dark field image which indicates a loss of crystallinity of the nanoparticle. This bulk like melting behavior for Ge in sapphire is attributed to the relatively large size of the nanocrystals. 


\section{CONCLUSIONS}

Isotopically controlled Ge nanocrystals were synthesized by selective ion implantation into a sapphire matrix. A bi-modal size distribution was observed with large faceted nanocrystals formed in the most damaged and strained area and smaller ones in the shoulder area. Larger stress was also observed from these Ge nanocrystals than from those embedded in a silica matrix. In-situ TEM heating experiments showed that the Ge nanocrystals in sapphire have a melting point close to the bulk Ge whereas the ones embedded in silica exhibit a large melting hysteresis around the melting point of bulk Ge.

\section{ACKNOWLEDGMENTS}

This work was supported in part by US NSF Grant Nos. DMR-0405472 and EEC-0085569, and by the Director, Office of Science, Office of Basic Energy Sciences, Division of Materials Sciences and Engineering, of the U.S. Department of Energy under Contract No. DE-AC03-76SF00098.

\section{REFERENCES}

1. T. Takagahara and K. Takeda, Phys. Rev. B 46 (15), 578 (1992).

2. K. S. Min, K. V. Shcheglov, C. M. Yang, and H. A. Atwater, Appl. Phys. Lett. 68 (18), 29 (1996)

3. H. Fukuda, S. Sakuma, T. Yamada, and S. Nomura, J. Appl. Phys. 90, 3524 (2001)

4. M. Takagi, J. Phys. Soc. Japan 9, 359 (1954).

5. A. N. Goldstein, C. M. Echer, and A. P. Alivisatos, Science 256 (5062), 1425 (1992).

6. Y. Wu and P. Yang, Appl. Phys. Lett. 77, 43 (2000)

7. H. Andersen and E. Johnson, Nucl. Instr. and Meth. B 106, 480 (1995).

8. Q. Xu, I. D. Sharp, C. Y. Liao, D. O. Yi, J. W. Ager III, J. W. Beeman, Z. Liliental-Weber, K. M. Yu, D. N. Zakharov, D. C. Chrzan and E. E. Haller, Mater. Res. Soc. Symp. Proc. 818, M11.17.1 (2004)

9. I. D. Sharp, D. O. Yi, Q. Xu, C. Y. Liao, J. W. Beeman, Z. Liliental-Weber, K. M. Yu, D. N. Zakharov, J. W. Ager III, D. C. Chrzan and E. E. Haller, Appl. Phys. Lett. 86, 063107 (2005)

10. F. Cerdeira, C. J. Buchenauer, F. H. Pollak, and M. Cardona, Phys. Rev. B 5, 580 (1972) 